\title{
Multilinguales
}

\section{Léon l'Africain à la « rencontre » de la Renaissance}

Leon the African at the encounter of the Renaissance

$$
\text { ليون الأفريقي في لقاء عصر النهضة }
$$

\section{Abdelkrim Benslim}

\section{(Q) OpenEdition}

\section{Journals}

Édition électronique

URL : http://journals.openedition.org/multilinguales/318

DOI : $10.4000 /$ multilinguales.318

ISSN : 2335-1853

Éditeur

Université Abderrahmane Mira - Bejaia

Référence électronique

Abdelkrim Benslim, «Léon l'Africain à la « rencontre » de la Renaissance », Multilinguales [En ligne], 8 | 2017, mis en ligne le 01 juin 2017, consulté le 10 décembre 2020. URL : http://

journals.openedition.org/multilinguales/318; DOI : https://doi.org/10.4000/multilinguales.318

Ce document a été généré automatiquement le 10 décembre 2020.

\section{cc) $(9)$}

Multilinguales est mise à disposition selon les termes de la Licence Creative Commons Attribution -

Pas d'Utilisation Commerciale - Pas de Modification 4.0 International 


\section{Léon l'Africain à la « rencontre » de la Renaissance}

Leon the African at the encounter of the Renaissance

$$
\text { ليون الأفريقي في لقاء عصر النهضة }
$$

\section{Abdelkrim Benslim}

1 En général, les historiens des idées considèrent les $\mathrm{XV}^{\mathrm{e}}$ et $\mathrm{XVI}^{\mathrm{e}}$ siècles comme période véritablement représentative de la Renaissance par excellence, et ce, sur tous les plans (Garin, 2002: 8). Et c'est justement durant cette période que Hassan el-Wazzan, un intellectuel arabo-andalou épris de découvertes et de voyages, et qui deviendra plus tard un diplomate, un traducteur ainsi qu'un éminent géographe, est fait prisonnier par un corsaire sicilien dont le nom est Pietro Bovadiglia lequel, pour expier ses crimes et autres torts de forban, l'offrit au pape Léon X en 1519. Ce dernier, conscient alors d'avoir capturé un "ambassadeur nord-africain " ayant de bonnes relations avec les Ottomans (Zemon Davis, 2007 : 70), ne le jeta pas en prison. Au contraire, il fut « installé dans une petite pièce, meublée d'un lit, d'une chaise et d'un coffre en bois, comme s'il s'agissait d'une modeste hôtellerie plutôt que d'une prison » (Maalouf, 1986 : 404). En fait, cette histoire de capture de Hassan était bien une aubaine pour Saint-Siège ; c'est d'ailleurs ce qui lui a été explicitement signifié par l'un des représentants du pape, un certain messire Francesco Guicciardini qui ne s'empêchait pas de lui avouer que le pirate espagnol ne s'était pas trompé de cible puisqu' «il savait quel type de Maure devrait être présenté au Saint-Père : un voyageur, un lettré [...] de surcroît, [...] un diplomate " (ibid. : 406). Dans le présent article, nous nous proposons de voir comment, dans son voyage-exil à Rome du début du XVI ${ }^{\mathrm{e}}$ siècle, Hassan appréhenda son contact avec les hommes lettrés et érudits de la Renaissance et comment il considéra sa captivité non pas comme une éprouvante tribulation mais plutôt comme péripétie fondamentale d'un périple initiatique qui allait lui ouvrir les yeux sur le vrai sens de la Renaissance et de l'humanisme en tant qu'éléments fondateurs de la modernité au pays de Léonard de Vinci et Michel-Ange. Nous verrons aussi comment l'érudit de Fès s'est vite accommodé de sa captivité pour en faire un séjour d'apprentissage, d'épanouissement et d'écriture. Dans le même temps, nous mettrons en exergue la 
valeur historique et cognitive de ses écrits et de son expérience existentielle. Pour ce faire, nous travaillerons sur Léon l'Africain, un roman écrit par Amin Maalouf en 1986 et qui incarne pertinemment la littérature viatique dans ses trois dimensions principales que sont la quête, le nomadisme et la scriptio. Au plan méthodologique, notre approche sera transdisciplinaire et du coup, empruntera aussi bien à la narratologie et à l'histoire des idées qu'à l'analyse du discours. On se permettra, par ailleurs, d'effectuer ça et là de petites incursions - surtout en termes d'intertextualité - dans les travaux de recherches sur le personnage référentiel Hassan el-Wazzan, connu sous le nom de Léon l'Africain.

\section{Léon l'Africain, une expérience viatique}

2 Mais commençons tout d'abord par saisir génériquement le corpus en question. Dans quelle mesure s'inscrit-il dans cette littérature dite viatique? Autrement dit, Léon l'Africain est-il ou non un récit de voyage? En fait, les critères de ce genre sont fluctuants et changent selon les théoriciens et les critiques. Evelyne Deprêtre, chercheure au département des études littéraires de l'université de Montréal, après avoir identifié certaines caractéristiques récurrentes dans la structuration du genre viatique, n'hésite pas à proposer la synthèse définitoire que voici :

Un récit de voyage est un récit factuel à vocation autobiographique - par sa référence à un voyage réalisé, [...] généralement écrit [à la première personne du singulier, le « je »]. Son auteur, pouvant recourir à plusieurs formes littéraires voire à leur hybridation, narre, [...] l'expérience viatique, en principe chronologique et géographique, de la rencontre qu'un sujet, portant le triple chapeau d'auteurnarrateur-voyageur, fait non seulement avec les espaces parcourus et l'altérité, mais aussi avec son lecteur implicite. (Deprêtre, $2011: 42$ ).

3 Cette définition, on le verra, correspond parfaitement à notre corpus. En effet, la factualité renvoie aux différents témoignages et événements relatés par le géographe Léon l'Africain lors de son voyage à travers la Méditerranée et qui, en fait, sont d'ordre autobiographique. Par ailleurs, le récit est écrit à la première personne du singulier comme on le voit dans l'incipit: «Moi, Hassan, fils de Mohamed le peseur, moi JeanLéon de Médicis, circoncis de la main d'un barbier et baptisé de la main d'un pape, on me nomme aujourd'hui l'Africain» (Maalouf, 1986:11). Le caractère protéiforme du roman, quant à lui, vient du fait qu'il épouse concomitamment plusieurs formes scripturales et génériques à la fois. En vue de ne pas excéder le cadre de notre étude, on dit tout simplement que cette pluralité est consubstantielle au genre viatique. Tout y est. La forme épistolaire : Ils firent parvenir à Fès des messages poignants comme dans cette lettre : Frères, si, à la chute de Grenade, nous avons failli à notre devoir d'émigrer, c'était uniquement faute de moyens, car nous sommes les plus pauvres et les plus affaiblis des Andalous (Maalouf, 1986: 146); la forme poétique: "Grenade, nulle cité ne te ressemble/ Ni en Égypte, ni en Syrie, ni en Irak/C'est toi la mariée/Et ces pays ne sont que ta dot (Ibid., p. 69) ; la forme sacrée : «Il y a sur la terre des signes, pour ceux dont la foi est solide. Il y en a aussi en vous-mêmes, ne les voyez-vous pas? Il y a dans le Ciel les biens qui vous sont destinés. Et aussi ce dont vous êtes menacés" [Coran] (Maalouf, 1986:127); la forme historique : «Le 25 mars 1527, le vice-roi de Naples, Charles de Lannoy, arriva à Rome, envoyé extraordinaire de l'empereur, pour conclure un accord» (Maalouf, $1986: 485$ ) ; la forme gnomique : "La route apporte la connaissance et la richesse, la montagne offre la protection et la liberté" (Maalouf, 1986 : 223). Sur un autre plan, celui de l'expérience viatique aussi bien chronologique 
que géographique, le récit de Léon l'Africain s'inscrit dans un itinéraire presque circulaire qui comprend l'Andalousie (Le Livre de Grenade), ensuite le Maroc, le Maghreb et l'Afrique profonde (Le Livre de Fès), Le Machreq (Le Livre du Caire) et enfin la rive italienne du bassin méditerranéen (Le Livre de Rome). Le récit, lui, s'échelonne sur presque une quarantaine d'années: de décembre 1488 (894 de l'hégire) à septembre 1527 (933 de l'hégire) ${ }^{1}$. Enfin, la lecture du roman nous apprend que Hassan y assume une triple instance: celle d'auteur (celui qui se charge de fixer son voyage-exil par l'écriture en signant son récit du nom de Hassan el-Wazzan), celle de narrateur (à travers le "je» de Hassan qui narre) et celle de voyageur (celui du personnage référentiel Hassan qui, tout en menant cette expérience de voyage, se livre à des observations et des témoignages de tous ordres.

A vrai dire, la question de la généricité n'est pas ce qui nous occupe dans cette analyse ; l'essentiel pour nous étant d'avoir identifié notre corpus en le catégorisant dans l'espace de la littérature de voyage ${ }^{2}$. Abordons présentement ce texte sur un autre plan, celui de l'expérience existentielle viatique que Hassan aurait vécue dans sa Rihla, mais dans l'aire européenne, c'est-à-dire à Rome. À ce titre, il convient de reconnaître que Les quatre carnets du roman (mais surtout le quatrième car s'inscrivant dans le sillage de la présente étude) nous livrent dans toute leur profondeur les aspects de ce voyageexil. Par aspects, nous entendons notamment la dimension exilique, ou mieux celle de captivité mais aussi et surtout les dimensions intellectuelle, culturelle et créatrice liées, elles, au séjour "forcé » que le géographe grenadin dût passer loin des siens. Dans quelle mesure donc Léon l'Africain, roman de l'académicien franco-libanais Amin Maalouf, traduit-il une expérience viatique, et de surcroît, des plus singulières au moment où le monde arabo-musulman commençait (dès avant 1492) à sombrer dans les abysses de l'Histoire ? Répondre à cette question revient en fait et de prime abord à appréhender l'état de captivité du personnage Hassan dans les multiples variantes du voyage. Autrement dit, les moments passés sous la férule du pape Léon $\mathrm{X}$ à Rome, ne peut-on pas légitimement les considérer comme un épisode déterminant dans ce grand périple de Léon autant que dans sa vie d'intellectuel captif ? D'abord, il est significatif de noter que cette période occupe un espace de grande importance dans le récit de voyage relaté par le captif. Pas moins de quatre-vingts pages s'échelonnant en effet sur une huitaine d'années : de 1519 à 1527. Il ne s'agit pas de quelque chose de négligeable. L'histoire nous apprend à ce sujet que les corsaires ne réservaient pas le même traitement ni le même sort à toutes leurs victimes. Les fortunés, les dignitaires ainsi que les intellectuels étaient plutôt une aubaine pour les ravisseurs. Ainsi, Bovadiglia, l'un des flibustiers les plus terribles de la Méditerranée, après avoir soupesé son captif,

sacrifia à l'habitude des pirates d'interroger leurs prisonniers afin de décider quels étaient ceux dont on pouvait espérer une rançon et quels étaient ceux qu'on vendrait comme esclaves; [en effet] il n'eut aucun mal à comprendre l'importance de l'ambassadeur nord-africain ni ses liens avec les Ottomans (Zemon Davis, 2007 : 70).

5 Et depuis ce jour-là, c'est-à-dire le 2 janvier1519, le long voyage du rahala Hassan dégénéra en captivité. Et partant, le périple allait prendre une autre trajectoire, une autre destinée. Tout allait changer en effet et Hassan de Rome ne sera plus Hassan de Grenade ni celui de Fès. En fait, et c'est le point qui nous intéresse dans cette étude, l'état de captivité n'avait en rien changé la vie du géographe ni amenuisé l'intrépidité de ses projets à vouloir faire de sa vie un voyage, même dans les moments difficiles de la détention qu'il allait vivre dans l'exil forcé au pays du Vatican. Là, une question 
corrélative surgit concernant cette nouvelle condition de vie liée à la privation de liberté. L'exil, physique et moral à la fois pour le cas du géographe arabo-andalou, n'est-il pas plutôt lié à l'expérience viatique qu'il avait entreprise des années auparavant ? La réponse est catégorique. Exil et voyage sont deux choses qui renvoient à la même condition d'éloignement, de mobilité et de déracinement. Ainsi, dans la préface d'un ouvrage intitulé Voyage, errance \& exil. Parcours existentiels, Abdelghani El Himani met en exergue cette impossibilité pour l'homme d'effacer les frontières entre voyage et exil :

Voyage, errance et exil [...] ! Peut-on vraiment distinguer entre ces processus ? [...]. Le voyage est le dénominateur commun, l'assise fondamentale sur laquelle ils reposent tous. Nulle errance n'est envisageable sans le recours au voyage, nul exil n'est envisageable sans périple qui le sous-tend. [...]. L'exil est un voyage forcé dans des contrées moins heureuses, un arrachement de l'espace enchanté de la complétude. (El Himani, $2016: 7$ ).

6 C'est dire toute la portée du voyage que comprend l'exil dans sa plénitude. On pourrait ainsi dire, sans risque d'exagération, que la captivité de Hassan el-Wazzan est un « destin » exilique qui s'inscrit profondément dans l'expérience viatique qui a fait de sa vie un ensemble d'escales quelquefois heureuses. Et par conséquent, tous les faits et gestes de ce personnage référentiel (dont l'écriture et les comportements culturels ou autres), pendant son séjour au Vatican, sont à inscrire dans le monde du voyage jusqu'à son retour à sa cité natale (Grenade) ou d'adoption (Fès). C'est ce qui a été en effet soulevé d'une façon pertinente par Ottmar Ette dans une étude publiée dans Romanische Studien. Pour ce critique et historien allemand, l'œuvre romanesque de l'académicien franco-libanais, obéit à cette logique qui privilégie le partir sur la patrie; point d'enracinement dans un territoire puisque la vie est, en définitive - et par définition -, issue du mouvement qu'est le voyage (Ette, 2015: 397-433): "On m'appelle [...] le Grenadin, le Fassi, le Zayyati, mais je ne viens d'aucun pays, d'aucune cité, d'aucune tribu. Je suis le fils de la route, ma patrie est caravane, et ma vie la plus inattendue des traversées" (Maalouf, 1986 : 11). Et comme la vie tout entière de Hassan el-Wazzan est un voyage, rien n'empêche d'avoir comme corollaire cette idée que le récit - tantôt sur un navire, tantôt dans le désert, tantôt en captivité, pour ne citer que ces cas de figure appartient à la littérature viatique. Enfin, un petit détail utile pour la présente étude, concernant le voyage chez Hassan : en fait, ce dernier est de deux ordres. Le premier, très terre à terre, renvoie à ce déplacement physique dans l'espace et le temps, une ambulation qui se fait à pied, sur le dos des montures, par voie maritime, etc. Le second, plus subtil, a plutôt trait à tout ce qui est abstrait, spirituel et intellectuel. Pour Hassan, le texte viatique en tant que scriptio et en tant qu'effort relevant de l'intellect et du logos, s'inscrit ainsi dans cette dernière. En un mot, il s'agit bel et bien d'une expérience existentielle viatique. Nous y reviendrons.

\section{2. À la rencontre de l'« homme de la Renaissance »}

7 Il n'est pas inutile de rappeler que ce sont les Italiens qui, les premiers, connurent la Renaissance. Florence, Rome et Venise furent le véritable foyer triangulaire de ce mouvement d'épanouissement littéraire, scientifique, artistique, architectural, etc. Et ce sont en fait ces trois Cités-Etats qui allaient imprimer, quelques années plus tard, un mouvement de dissémination des différentes idées fondatrices de la Rinascita sur l'Espagne, les pays du nord (Allemagne, Hollande), la France et l'Angleterre. Dans le 
même temps, à Grenade, nombreux étaient ceux qui avaient pris le chemin de l'exil. La Reconquista, quant à elle, exterminait les dernières populations musulmanes restées sur place en les traduisant devant les fameux tribunaux de l'Inquisition soit pour les forcer à adopter le christianisme, soit encore pour leur infliger les pires tortures en cas de suspicion ou de refus de changer de religion.

Cet épisode sera longuement narré, et en détail, par Hassan el-Wazzan dans son récit de voyage. Et c'est précisément pendant ces années renaissantes que Hassan se fit capturer par l'un des plus redoutables corsaires du bassin méditerranéen et ensuite livré à titre d'« offrande votive » au pape Léon X (Zemon Davis, 2007 : 70). Et là, le géographe arabe n'avait pas d'autre choix que de se voir, après plus d'un mois de captivité, baptisé par le pape lui-même. Celui-ci lui donnera en effet le nom de Jean Léon, le nom de son protecteur et désormais tuteur, le grand pape de la Chrétienté et le premier homme du Vatican : "Il m'avait donné ses deux prénoms, Jean et Léon, ainsi que le nom de sa prestigieuse famille, les Médicis, le tout avec pompe et solennité, le 6 janvier 1520, un vendredi, dans la nouvelle basilique Saint-Pierre » (Maalouf, $1986: 412$ ). Il faut reconnaître à ce titre que ce baptême allait octroyer un statut singulièrement privilégié à Hassan. Et depuis ce jourlà, tout allait changer dans la vie de ce dernier, à commencer par son nom...et sa religion. La captivité et ce qui s'ensuivit donc coïncidaient curieusement avec les événements alors d'actualité. En effet, beaucoup de faits avaient émaillé l'époque de Hassan/Léon : d'abord, des faits liés à la décadence, à la chute du monde arabe à travers l'effondrement du dernier royaume de l'Andalousie, en l'occurrence Ghernata. Ensuite, une nouvelle civilisation était en train de faire son chemin après la découverte des deux Amériques par Christophe Colomb en 1492. D'ailleurs, en matière d'historiographie, cette époque charnière est appelée Año crucial (année cruciale), une année (et plus qu'une année, une époque) qui représentait à la fois la réhabilitation de l'hégémonie catholique après la reddition de Boabdil (le dernier roi Abû Abdil-lah, fils d'Abu IHasan Ali Muhammed XI, né à Grenade en 1459) et l'essor civilisationnel de l'Europe émergente, subséquent (entre autres faits historiques, bien évidemment) à la découverte historique du navigateur Colomb.

Non loin de l'Espagne et de la Reconquista, en Italie, l'«homme de la Renaissance " commençait à défrayer la chronique. Mais avant de voir comment se tissait la relation de Léon/Hassan avec les artistes, les savants et autres intellectuels italiens de l'époque, il nous faut expliquer ce que nous venons de mettre entre guillemets. Il s'agit en réalité de cet « homme » qui, dès la fin effective du Moyen Âge et le début de la Renaissance, notamment en Italie, décida de rompre définitivement avec la conception traditionaliste de l'homme, une conception d'obédience religieuse et superstitieuse par excellence. C'est surtout cet homme renaissant reflétant et concrétisant cette » image durable de la Renaissance comme moment décisif de la civilisation italienne» (Garin, 2002 : 10). C'est également cet homme dont l'historien des idées Eugenio Garin - à la suite des deux historiens, Jacob Burkhardt (l'un des pères fondateurs du paradigme « homme de la Renaissance ») et Agnès Heller - expliquera les traits en ces termes: "L'homme nouveau, l'homme moderne, était un homme qui se faisait, qui se construisait, et qui était [surtout] conscient de cette création. C'était, précisément, l" homme de la Renaissance" " (Garin, 2002 : 12). Ceci étant, la question de connaître la nature de la relation du protagoniste éponyme du roman Léon l'Africain aux « homme[s] de la Renaissance » est d'une importance et d'une légitimité incontestables. En effet, de quelle manière cette relation s'était-elle construite? Etait-ce une relation de dominé /dominateur? Et de 
quelle manière Hassan El-Wazzan appréhendait-il ce rapport à l'Autre? Dans son ouvrage Un voyageur entre deux mondes, Nathalie Zemon Davis n'hésite pas à développer l'analyse de cette relation dans deux chapitres interdépendants, respectivement intitulés «Vivre en terre de guerre » et «Écrire en Italie ». L'appellation du premier chapitre est d'une pertinence manifeste dans la mesure où d'une part, l'auteure explicite la vie du captif de la guerre entre Chrétiens et Musulmans et qui n'était en définitive que l'autre face des Croisades de l'époque, et d'autre part, une manière de dire la condition d'assujettissement et d'aliénation dans laquelle dût vivre ce voyageurgéographe-écrivain si intrépide des mois durant, loin des siens, loin de sa culture, loin de sa terre, loin du " muezzin ». Une fois livré au château Saint-Ange, Hassan ne fut pas incarcéré comme beaucoup d'autres «ennemis » de la Chrétienté du Vatican. À son étonnement, à aucun moment, il ne fut victime de sévices; tout au contraire, il «fut suffisamment bien traité pour qu'on lui prête des manuscrits en arabe de la Bibliothèque vaticane moins d'un mois après son arrivée, en 1518 " (Zemon Davis, 2007 : 72). Ceci, Léon le reconnaîtra et le narrera dans son récit. Ainsi, pour le pape Léon X s'adressant au diplomate de Fès lors du baptême, il s'agit bien d'une vérité établie auprès du château de Città del Vaticano: "un homme d'art et de connaissance est toujours le bienvenu auprès de Nous, non comme serviteur, mais comme protégé. Il est vrai que votre arrivée dans cette demeure a eu lieu contre votre gré par des moyens et que Nous ne saurions approuver " (Maalouf, 1986 : 407). Lors de l'entrevue entre Hassan et celui qui allait devenir son protecteur, l'intellectuel grenadin comprit bien que si le souverain pontife lui avait accordé un échange (et non pas un interrogatoire auquel on soumet d'ordinaire un captif), c'était justement là une manière d'être chanceux ; c'était aussi un signe de réconfort de la part de l'homme puissant de Rome. C'est ainsi qu'en homme avisé et pondéré, il accepta le fait accompli et décida de s'accommoder de la nouvelle situation : vivre à Rome, vivre avec les hommes de Rome, changer de mode de vie. Mais pour combien de temps? Ce nouveau tournant dans la vie du voyageur captif, désormais tombé dans l'infortune, constitue un nouveau mode existentiel qui allait lui dicter de nouvelles contraintes en matière de comportement, d'attitude et de pensée. En lui accordant la libre circulation dans l'enceinte du château, le pape voulait surtout lui signifier, dès le début de sa captivité, qu'il bénéficiait d'une grande considération auprès de l'Église et que par conséquent il ne fallait pas le décevoir : "Quel que soit mon sentiment, je ne pus trahir mon protecteur " (Maalouf, 1986: 411); au bout du compte, le pape Léon X était bel et bien cet "homme qui [1]'avait pris sous ses ailes et qui [1]e traitait désormais comme s'il était [s]on géniteur» (ibid.: 412). Dans une seconde entrevue avec lui, tout un programme allait lui être offert en guise d'apprentissage. En effet, un homme de la trempe de Léon ne pouvait faire intégrer le cercle de Saint-Ange ni entrer en contact avec les savants, les artistes et les érudits italiens sans passer par un certain apprentissage, une certaine formation initiatique en vue d'acquérir le viatique qu'il faudra pour vivre dans la première cité européenne qui connut la Renaissance : "un évêque allait m'apprendre le latin, un autre le catéchisme, un troisième l'évangile ainsi que la langue hébraïque; un prêtre arménien me donnera chaque matin un cours de turc " (Maalouf, 1986 : 410). En contrepartie, Hassan devait apprendre la langue arabe à sept élèves. Mais, le plus curieux, c'était lorsque celui-là apprit qu'il allait être rémunéré : «pour ce travail, je percevrais un salaire d'un ducat par mois » (Maalouf, $1986: 411$ ). Un salaire pour un captif? Et au cœur de la Cour du Vatican? Une véritable gageure. Presque douze mois après, le contrat fut bien honoré : «Mon année de captivité fut donc sans peine pour le corps et fort profitable pour l'esprit » (Maalouf, 1986 : 410). Là, il est sans doute important 
de souligner dès maintenant un fait pas du tout anodin : en apprenant à fond le latin, l'hébreu et le turc, Jean Léon ne fit, après tout, que maîtriser les trois grandes langues de la civilisation méditerranéenne en plus de l'arabe qui, lui, était sa langue maternelle, la langue de ses ancêtres, la langue de sa culture. Ce détail, on l'explorera une fois qu'on aura abordé le rapport du géographe grenadin à la traduction.

Pour ce qui est de la rencontre du "nouveau Roi mage» (Maalouf, 1986: 413) qu'était Hassan, avec les érudits et les artistes de Rome, tout avait commencé lors de sa douce réclusion au château pontifical. Celle-ci lui était bénéfique sur tous les plans: connaissances de tous ordres, langues vivantes, traduction, relations intellectuelles et culturelles : "D'un jour à l'autre, je sentais mes connaissances s'élargir, non seulement dans les matières étudiées, mais également par le contact avec mes professeurs, ainsi qu'avec mes élèves, deux prêtres aragonais, deux Français, deux Vénitiens, un Allemand de Saxe " (Maalouf, 1986 : 410). Voyageur invétéré, il n'aimait pas dissocier sa captivité de ses projets de découvertes géographiques et de son expérience existentielle viatique. Au lendemain de son baptême, en allant se promener en dehors de l'enceinte du château, il n'oubliera pas, par exemple, de consigner dans le Livre de Rome :

Je ne gardais plus de ma captivité ni amertume ni ressentiment. Quelques semaines de lourdes chaînes, quelques mois de servitude douce, et voilà que j'étais redevenu voyageur, créature migrante, comme dans tous les pays où j'avais séjourné et obtenu, pour un temps, plaisirs et horreurs. Que de rues, que de monuments, que d'hommes et de femmes j'avais soif de découvrir. (Maalouf, $1986: 415$ )

11 Dans son esprit, aller à la rencontre des autres, surtout lorsque ceux-ci étaient les témoins d'événements importants liés à la Renaissance, exigeait avant tout la mise au diapason de ses facultés cognitives et intellectuelles ainsi que sa culture avec les avancées et autres bons usages de l'époque. C'est d'ailleurs dans cette optique qu'il apprit les langues prédominantes dans le bassin méditerranéen et qui, en réalité, représentaient les langues de transmission du savoir humain, mais aussi de transactions commerciales et juridiques et de traités diplomatiques, ainsi que de négoces maritimes. Aller au contact d'autres cultures et d'autres civilisations, voilà une finalité que s'est assignée Léon tout au long de ses voyages. Mais tout cela présupposait cette grande habileté d'être polyglotte en vue de pouvoir fructifier tous ces contacts humains et culturels. Lors de la cérémonie de baptême, beaucoup d'érudits, de poètes, d'artistes et autres dignitaires étaient présents : certains d'entre eux étaient les acteurs réels de la Haute Renaissance artistique tel que "Raphaël d'Urbino, le divin Raphaël, comme l'appelaient les admirateurs de son art» (Maalouf, 1986: 412), une figure universellement emblématique en matière d'architecture, de Beaux-Arts et de peinture en particulier. En fait, Youhanna al-Assad, "la signature qu'on peut voir au bas des ouvrages que [Léon/Hassan a] écrits à Rome et à Bologne » (Maalouf, 1986 : 414) l'avait deux fois rencontré. Certes, les rencontres étaient rapides mais elles avaient été consignées dans sa relation de voyage. Il lui avait même promis d'aller voir son atelier mais la mort fut plus rapide. Selon l'historienne américaine Zemon Davis, à Rome, Youhanna ne servit pas seulement le chef spirituel de la chrétienté Léon X mais aussi le cardinal Gilles de Viterbe, le prince Alberto Pio de Carpi, le bibliothécaire du Vatican Jérome Aléandre, le chancelier du pape Jules de Médicis. A ceux-là s'adjoignaient également des humanistes d'Italie comme Angelo Colocci, Paul Jove, Pierio Valeriano et des savants étrangers venus à Rome s'inspirer des «Lumières » de la Renaissance, tels que Eilas bar Abraham, Élie Lévita et Jacob Mantino. «Avec eux, Hassan el-Wazzan a eu des conversations savantes et des échanges d'idées de haut niveau » (Zemon Davis, 2007 : 223). En 
réalité, ces rencontres dépassaient de loin ce qui occupait et préoccupait le sens commun. Chanceux qu'il était après avoir découvert la Renaissance européenne avant ses semblables Arabes dans les diverses contrées du monde musulman, il était devenu cet intellectuel oriental cosmopolite. Ces rencontres, cette ouverture aux autres et ce sens de véritable cosmopolitisme, avaient fait de lui une personnalité intellectuelle jouissant d'une autorité authentique et d'un magistère indéniable. Il «devint en Italie un érudit prisé et finalement un auteur au statut d'expert " (loc. cit.). Et ce talent, paradoxe notoire de l'Histoire, s'était cristallisé et raffermi dans la cité vaticane et non chez les siens, une posture rappelant à bien des égards la fameuse expression biblique « Nul n'est prophète en son pays ».

Dans le même esprit de la rencontre avec les hommes de la Renaissance, il y a lieu de faire ici allusion à quatre célèbres érudits français qui étaient allés en Italie à l'époque même où Léon séjournait à Rome. Il s'agit de Michel de Montaigne (1533- 1592), François Rabelais (1483-1553), Joachim de Bellay (1522-1560) et de Jean Bodin (1529 1596). Si les trois premiers sont connus (surtout à travers le programme du collège et de l'université), le dernier l'est moins. Celui-ci

est à tous les sens du terme un homme de la Renaissance. Très cultivé, il est philosophe, historien, juriste et même théologien. [Il est l'auteur du] concept de souveraineté légitime désormais le pouvoir en donnant à la volonté humaine une fonction fondatrice en politique (Chanteur, 1991 : 283-294).

Selon une étude menée par le philosophe Ali Benmakhlouf dans le cadre d'une perspective de l'Unesco pour la promotion du dialogue entre les cultures, les deux érudits «Bodin, Montaigne reprennent [...] des exemples de Léon [1'Africain] (Benmakhlouf, 2010 : 17-29); ce qui revient à dire que même s'il n'y a pas eu une rencontre physique entre ces érudits français - véritables « hommes de la Renaissance » - et Hassan, rien ne nous interdit de dire qu'il y a eu rencontre, sinon livresque, du moins intertextuelle. Cette intertextualité, surtout lorsqu'elle s'inscrit dans la contemporanéité (c'est-à-dire au siècle du pape X), devenait parfois inévitable au XVI siècle. C'est ce qui fera parler Frank Lestringant, à juste titre, "d'étonnants phénomènes d'intertextualité découl[a]nt de [la] continuité régnant entre les divers genres d'écriture " (Lastringant, 2005 : 759-769). Cependant, même si Rabelais ne s'est pas inspiré de Hassan, "considérons, note l'historienne américaine Zemon Davis, plutôt leur vie et leur manière de penser et d'écrire à leur époque, dans laquelle on peut déceler de frappantes similitudes par-delà le fossé qui pouvait les séparer " (Zemon Davis : 2007 : 303). Il n'est pas dans notre intention de multiplier les exemples afin de montrer ces traits de ressemblance. Le plus important pour la présente étude, c'est de faire allusion aux différents contacts qui auraient pu avoir lieu entre ces érudits et Léon l'Africain. De toutes les façons, tous - y compris Léon l'Africain - étaient des « hommes de la Renaissance».

\section{La description, un récit viatique écrit dans la cité papale}

Les neuf années que Hassan/Léon passa en Italie ont été fort bénéfiques, tant au plan de l'acquisition des connaissances savantes que celui de l'écriture. Le fait d'avoir " rencontré » la Renaissance et ses hommes n'était nullement une escale dans le long périple d'un intellectuel aguerri mais tout juste une pause d'apprentissage, de découverte de nouveaux horizons et d'épanouissement qui allaient lui ouvrir les yeux 
sur un monde qui commençait sérieusement à se dépoussiérer, tout en lui «étendant aussi le sentiment de ses possibilités intellectuelles" (Maalouf, 1986: 260). Cumulant connaissances arabes acquises surtout à la mosquée Karaouine et la médersa Bou Inaniya au Maroc, et nouveau savoir né de la Renaissance italienne tout en brassant des disciplines de tous ordres à Tombouctou, en Egypte, en Turquie, à La Mecque, entre autres escales méditerranéennes et africaines, il finit par devenir un véritable polymathe. Ses compétences étaient celles d'un géographe averti, d'un faqih (jurisconsulte) malékite ouvert aux autres écoles du figh, d'un diplomate négociant commercial réaliste, d'un administrateur ayant déjà la savoir-faire de la cour royale du Maroc, d'historien et sociologue rahala qui sait tout consigner dans son récit de voyage, d'un commentateur de texte religieux et enfin d'un traducteur qui a intelligemment su s'intégrer dans le cercle des adeptes d'Erasme dont l'objectif humaniste était d'offrir des traductions à l'humanité tout entière. Il nous faut souligner le caractère prolifique des œuvres de Léon l'Africain. Certes, le texte d'Amin Maalouf ne cite pas assez en matière de scriptio, mais il évoque à titre d'exemple Description de l'Afrique (en fait Libro de la Cosmographia et Geographia de Affrica), un livre écrit dans la langue des Italiens et dans lequel «son auteur fait un constant va-et-vient entre Europe et Afrique, entre différentes cultures et gouvernements d'Afrique, entre islam et christianisme" (Maalouf, 1986: 129). Oumelbanine Zhiri, l'une des grands spécialistes de la biographie et de l'œuvre de Hassan el-Wazzan, montre dans une étude, qui lui est consacrée, que le livre en question venait combler le vide des connaissances en matière de géographie, d'histoire, de philosophie d'idées dans une région, jusque-là, inconnue des Européens, en l'occurrence l'Afrique du Nord et l'Afrique sub-saharienne. Il est d'ailleurs fort significatif, d'après cette chercheure, de savoir que c'est Giovanni Battista Ramusio, luimême géographe et éditeur italien, qui le dit en guise de témoignage d'un spécialiste connu pour ses travaux dans l'Europe de la Renaissance - et sans ambages - dans la première édition de Geographia de Affrica en 1550 : "Je suis absolument certain qu'en lisant ce livre et en considérant ce qu'il contient et ce qui y est exposé, ils [les "seigneurs et princes »] que les informations qu'ils possèdent sont, auprès de celles-ci, brèves et insuffisantes et de peu de valeur » (Zhiri, 1991 : 13). Mais le témoignage le plus important et le plus remarquable est peut-être celui de Charles-Henri-Auguste Schefer. Célèbre orientaliste, mais aussi écrivain, traducteur, historien et géographe français, il était membre de l'Académie des Inscriptions et Belles-Lettres, l'une des cinq académies de l'Institut de France. Dans son Recueil de Voyages et de Documents pour servir à l'Histoire de la géographie depuis le XIII ${ }^{e}$ jusqu'à la fin duXVIe siècle (encyclopédie de récits de voyages annotés de quinze volumes), il mit en valeur l'exactitude ainsi que la pertinence des études et des découvertes géographiques qui ont été faites par le « lettré musulman »:

Il était réservé à un lettré musulman d'origine espagnole de nous fournir, dès la première moitié du $\mathrm{XVI}^{\mathrm{e}}$ siècle, des notions exactes sur le Maghreb, sur les principaux Etats musulmans du nord de l'Afrique, ainsi que sur les contrées qui les avoisinent. Toutes les études et toutes les découvertes faites dans ces derniers temps ont permis de constater la valeur des renseignements qu'il nous a fournis et tous les géographes qui, durant les trois derniers siècles, ont fait de l'Afrique l'objet de leurs investigations ont exclusivement consulté l'ouvrage publié par lui [...]. L'ouvrage de Léon l'Africain fixa dès son apparition l'attention des savants. Une première édition en avait paru en 1550 dans le Recueil des Navigationi e Viaggi de Ramusio ; elle fut suivie d'une seconde quatre années plus tard, et c'est sur celle-ci que furent faites, la traduction latine par [Jean] Fleurian, et la traduction française par Jean Temporal [...]. Les savants qui, au XVII ${ }^{\mathrm{e}}$ siècle, se sont occupés de la

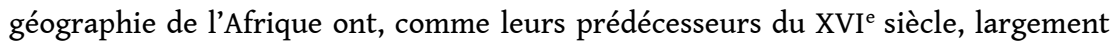


puisé dans l'ouvrage de Jean Léon l'Africain. Je citerai tout d'abord Gramaye [JeanBaptiste, historien, diplomate et géographe]. Il s'appuie sur l'autorité de Jean Léon l'Africain dans le chapitre où il traite de l'origine de la langue parlée par les habitants du Maghreb. (Schefe et Cordier, 1881 : VI).

Il est également significatif à ce sujet de voir l'historien finlandais Pekka Masonen l'un des spécialistes notoires de l'ancien empire soudanais et du commerce transsaharien - évoquer la valeur de la Description de Léon dans une Europe qui, elle, était encore indigente en matière de géographie, de prosopographie et de sociologie africaines :

Depuis sa première publication en 1550 , et pendant presque trois siècles, la Descrittione dell'Africa d'al-Hasan b. Muhammad al-Wazzan - mieux connu sous le nom de Léon l'Africain - a constitué pour les lecteurs européens la source la plus importante sur la géographie et l'histoire de l'Afrique soudanaise. Sa popularité persistante s'explique par l'absence de travaux équivalents avant le début de l'exploration européenne du continent africain à la fin du XVIII ${ }^{\mathrm{e}}$ siècle [...]. Quels que soient les mérites de Léon dans d'autres domaines, son importance dans l'historiographie de l'Afrique soudanaise se fonde essentiellement sur le fait qu'il a été le premier à introduire le sujet auprès des savants européens (Masonen, 2006 : 71-81).

16 Par ailleurs, il convient de souligner le caractère universel de l'écriture de Hassan elWazzan. Nous avons évoqué surtout La Description pour sa célébrité dans le monde occidental depuis la Renaissance. Mais d'autres ouvrages non moins importants ont été écrits par ce " voyageur entre deux mondes ». Afin de montrer que ce dernier était un érudit dont l'écriture débordait largement sa vocation qu'était la géographie politique, et loin de nous toute prétention à l'exhaustivité, nous nous suffirons d'énumérer quelques unes de ses œuvres, mais toutes rédigées lors de ses pérégrinations ; ce qui les inscrit et les définit génériquement dans la sphère des récits de voyage puisque c'est lors de son dernier voyage notamment, donc dans sa captivité ensuite son libre séjour à Rome, qu'elles ont été rédigées. Les titres sont donnés, ici, en italien, langue d'écriture du polymathe arabo-andalou: De Arte Metrica Liber, De Viris quibusdam Illustribus apud Arabes $^{3}$, De Viris quibusdam Illustribus apud Hebraeos ${ }^{4}$, Libro de la Cosmographia [sic] et Geographia de Affrica. Il y en a bien sûr d'autres traitant notamment du figh et de la traduction. Il convient toutefois de savoir une chose. La plupart de ces ouvrages (et d'autres qu'on trouve encore sous forme de manuscrits ${ }^{5}$ dans des bibliothèques italiennes et espagnoles) n'ont pas fait l'objet de traduction ni même de consultation "sérieuse " de la part de chercheurs aussi bien en Europe qu'au Maghreb. Pour ne donner qu'une seule illustration sur cet amer constat, nous donnerons l'exemple du dictionnaire arabe-hébreu-latin dans lequel la collaboration de Youhanna al-Assad est attestée par les grands traducteurs et autres lexicographes de l'époque. A ce titre, David Kaufmann, le biographe de Jacob Mantino, après avoir cité nommément Hassan, revient sur La Description et sur ledit dictionnaire :

Son ouvrage sur l'Afrique, auquel on a dû pendant longtemps les seules notions concernant cette région du monde si peu connue, lui valut le surnom honorifique de l'Africain. C'est pour Jacob Mantino, son ami juif ${ }^{6}$ pour le savant professeur et célèbre médecin, comme il rappelle dans sa dédicace arabe, que Léon écrivit à Bologne, 1524, son vocabulaire arabe-hébreu-latin qui a été conservé parmi les manuscrits arabes de l'Escurial (Kaufmann, $1893: 12$ ).

17 Ainsi, et pour ne s'en tenir qu'à cet exemple ô combien significatif, pour le protagoniste-narrateur de Léon l'Africain, « [il s]'engage[a] à fournir les parties arabe et hébraïque sur la base d'une longue liste de mots latins » (Maalouf, 1986 : 459). 
A vrai dire, à travers notre analyse, nous avons mis en relief l'appartenance effective de Hassan/Léon à la pléiade d'érudits qui avaient collaboré à l'émergence de la Renaissance. Sa vie, qui était un vrai récit de voyage, est faite de hauts et de bas. Cependant, en polymathe sagace et sage, il a su faire de sa captivité, et partant de son exil forcé, un moment très profitable à l'esprit et à la créativité. Son singulier sens d'accommodation lui a valu d'être estimé, ou du moins respecté de ses semblables européens. Son séjour à Rome s'inscrit dans son long projet viatique. L'Italie ne fut pour lui qu'une escale parmi d'autres. Et ses pérégrinations effectuées en Afrique sahélienne, en Afrique du Nord, en Turquie, au Hijez (Arabie) en Italie, lui avaient rassemblé les ingrédients précieux et nécessaires pour écrire sa vie, car sa vie n'était autre qu'un récit de voyage, un livre à lire, un livre écrit par un « homme de la Renaissance ».

\section{BIBLIOGRAPHIE}

BENMAKHLOUF A., « Le concept de civilisation », dans BENMAKHLOUF A. et al., La civilisation arabo-musulmane au miroir de l'universel : perspectives philosophiques, Unesco, Paris, 2010. Disponible sur < unesdoc. Unes co.org/images/0019/001917/191746f.pdf>.

CHANTEUR J., « La loi naturelle et la souveraineté chez Jean Bodin », in « Théologie et droit dans la science politique de l'État moderne ». Actes de la table ronde de Rome (12-14 novembre 1987) Rome : École Française de Rome, 1991. pp. 283-294. Disponible sur <http://www. persee.fr/doc/ efr_0000-0000_1991_act_147_1_4176>.

DEPRÊTRE E., « Le récit de voyage : quête historique et définitoire, la préoccupation de l'écrivain ", Mémoire de maître ès arts, Rimouski (Québec), 2011. Disponible sur < semaphore.uqar.ca/530/1/Evelyne_Depretre_fevrier2011.pdf>.

ELHIMANI A. (préface) dans SEMLALI M. (dir.), Voyage, Errance \& Exil, Université Sidi Mohamed Ben Abdellah, Fès, 2016.

GARIN E. (dir.), L'homme de la Renaissance, Seuil, 2002.

KAUFMANN D., « Jacob Mantino. Une page de l'histoire de la Renaissance », in La Revue des études juives, tome XXVll, Paris, 1893. Disponible sur <https://archive.org/details/bub_gb_al0-

AAAAYAAJ>.

LANNI D., « État présent des recherches en français sur la littérature des voyages », in Annali d'Italianistica, vol. 21, pp. 483-499, 2003, Arizona (USA). Disponible sur <http://www.jstor.org/ stable/24009878>.

LASTRINGANT F., « Renaissance ou XVI e siècle ? Une modernité étranglée », in Revue d'histoire littéraire de la France, Paris, 2002. Disponible sur <http://www.cairn.info/revue-d-histoirelitteraire-de-la-france-2002-5-page-759.htm>.

MAALOUF A., Léon l'Africain, Lattès, Paris, 1986.

MASONEN P., «Léon l'Africain et l'Historiographie de l'Afrique Soudanaise », in Studia Islamica, $\mathrm{N}^{\circ}$ 102/103, pp. 71-89, Maisonneuve \& Larose, Paris, 2006. Disponible sur <http://www.Jstor.org/ stable/20141084>. 
SCHEFE Ch., CORDIER H., Recueil de Voyages et de Documents pour servir à l'Histoire de la géographie depuis le XIII ${ }^{e}$ jusqu'à la fin du XVI siècle, Ernest Leroux, Paris, 1881. Disponible sur < http:// www.archive.org/details/descriptiondelaf01leoa>.

ZEMON DAVIS N., Léon l'Africain. Un voyageur entre deux mondes, Payot, Paris, 2007.

ZHIRI O., L'Afrique au miroir de l'Europe : fortunes de Jean-Léon l'Africain à la Renaissance, Droz, Genève, 1991.

\section{NOTES}

1. Pour la question de généricité de l'écriture romanesque d'Amin Maalouf, consulter notre thèse de doctorat (non publiée) : BENSELIM Abdelkrim, La figure de l'intellectuel dans l'œuvre romanesque d'Amin Maalouf, Université d'Oran, 2013.

2. En fait, il n'y a pas de récit de voyage entièrement « autonome ». Cette assertion est confortée par les conclusions d'un spécialiste de la littérature viatique, en l'occurrence François Moureau : «Le pur récit de voyage est un accident de la littérature » (Lanni, 2003 : 483-499).

3. Sur quelques hommes illustres parmi les Arabes.

4. Sur quelques hommes illustres parmi les Juifs.

5. Ces manuscrits - et d'autres - se trouvent actuellement dans la Biblioteca Mediceas Laurenziana à Florence, la Biblioteca Nazioanale Centale à Rome, la Biblioteca Ambrosiana de Milan, la Biblioteca Estense Universitaria de Modène, la Real Biblioteca del Escorial en Espagne, l'Archivio Segreto Vaticano et la Biblioteca Apostolica Vaticana au Vatican.

6. Jacob Mantino: savant et médecin juif italien. Auteur de beaucoup de traductions scientifiques, surtout d'hébreu en latin. Co-auteur, avec Hassan el-Wazzan, du dictionnaire arabehébreu-latin.

\section{RÉSUMÉS}

Cet article a pour objet d'étude le récit de voyage de Hassan el-Wazzan, alias Jean-Léon l'Africain ; il se propose de montrer comment ce personnage mi-fictif mi-réel peut être considéré comme un «homme de la Renaissance » et comment, dans sa captivité à Rome, il a pu transformer son «séjour» carcéral en un moment d'apprentissage de nouvelles connaissances, de réflexion approfondie sur les problèmes de l'humanité et de créativité scripturale. Véritable quête initiatique, cet exil forcé lui permet, grâce à sa sagacité et à sa sagesse, d'entrer de plain-pied dans la Renaissance italienne. Nous aurons à appréhender l'expérience d'éloignement de Léon des siens comme un véritable récit viatique à lire aussi bien en Europe qu'au Maghreb.

The purpose of this article is to study the travel narrative of Hassan el-Wazzan, alias Jean-Léon l'Africain; he proposes to show how this half -real and half-fictional character can be considered as a "man of the Renaissance" and how, in his captivity in Rome, he was able to transform his prison stay into a moment of learning new knowledge, reflection and scriptural creativity. A veritable initiatory quest, this forced exile enabled him, thanks to his sagacity and his wisdom, to enter fully into the Italian Renaissance on the same level. We shall have to apprehend the 
experience of Leon's departure from his own as a true story to be read in both Europe and the Maghreb.

INDEX

Mots-clés : Léon l'Africain, Hassan el-Wazzan, récit viatique, homme de la Renaissance, Descrittione dell'Africa

ليون الإفريقي, حسن الوزان, قصة سفر, رجل عصر النهضة, وصففهرس الكلمات المفتاحية:

أفريقيا

Keywords : Leo the African, Hassan el-Wazzan, travel story, man of the Renaissance, Descrittione dell'Africa

\section{AUTEUR}

ABDELKRIM BENSLIM

Centre universitaire Belhadj Bouchaib

Aïn Témouchent 